\title{
Prognostic value of total number of lymph nodes retrieved differs between left-sided colon cancer and right-sided colon cancer in stage III patients with colon cancer
}

Lin Yang ${ }^{1,2,3+}$ (D), Zhenchong Xiong ${ }^{1,2,3 \dagger}$, Qiankun Xie $e^{1,2,3 \dagger}$. Wenzhuo He $e^{1,2,3}$, Shousheng Liu $u^{1,2,3}$, Pengfei Kong ${ }^{1,2,3}$, Chang Jiang ${ }^{1,2,3}$, Guifang Guo $0^{1,2,3^{*}}$ and Liangping Xia ${ }^{1,2,3^{*}}$

\begin{abstract}
Background: The consensus is that a minimum of 12 lymph nodes should be analyzed at colectomy for colon cancer. However, right colon cancer and left colon cancer have different characteristics, and this threshold value for total number of lymph nodes retrieved may not be universally applicable.

Methods: The data of 63,243 patients with colon cancer treated between 2004 and 2012 were retrieved from the National Cancer Institute's Surveillance, Epidemiology, and End Results database. Multivariate Cox regression analysis was used to determine the predictive value of total number of lymph nodes for survival after adjusting for lymph nodes ratio. The predictive value in left-sided colon cancer and right-sided colon cancer was compared. The optimal total number of lymph nodes cutoff value for prediction of overall survival was identified using the online tool Cutoff Finder. Survival of patients with high total number of lymph nodes $(\geq 12)$ and low total number of lymph nodes $(<12)$ was compared by Kaplan-Meier analysis.

Results: After stratifying by lymph nodes ratio status, total number of lymph nodes $\geq 12$ remained an independent predictor of survival in the whole cohort and in right-sided colon cancer, but not in left-sided colon cancer. The optimal cutoff value for total number of lymph nodes was determined to be 11. Low total number of lymph nodes $(<11)$ was associated with significantly poorer survival after adjusting for lymph nodes ratio in all subgroups except in the subgroup with high lymph nodes ratio (0.5-1.0).

Conclusions: Previous reports of the prognostic significance of total number of lymph nodes on node-positive colon cancer were confounded by lymph nodes ratio. The 12-node standard for total number of lymph nodes may not be equally applicable in right-sided colon cancer and left-sided colon cancer.
\end{abstract}

Keywords: Total number of lymph nodes, Lymph node ratio), Left colon cancer, Right colon cancer, Overall survival (OS)

\section{Background}

In patients with colon cancer' survival is independently associated with the number of lymph nodes analyzed at the time of colectomy [1]. This is true in both nodepositive and node-negative disease, indicating that the benefit is not solely due to upstaging and administration

\footnotetext{
* Correspondence: guogf@sysucc.org.cn; xilp@sysucc.org.cn

${ }^{\dagger}$ Equal contributors

${ }^{1}$ Sun Yat-sen University Cancer Center, 651 Dongfeng Road east, Guangzhou

510060, China

Full list of author information is available at the end of the article
}

of adjuvant therapy [1-3]. The American Joint Committee on Cancer recommends that a minimum of 7-14 lymph nodes be examined at colectomy to avoid understaging $[4,5]$. The World Congress of Gastroenterology (1990) consensus was that at least 12 lymph nodes should be examined to ensure complete resection and adequate staging $[1,6,7]$. However, prognosis also depends on the status of regional lymph nodes, which is a major determinant of the need for adjuvant therapy.

Right-sided colon cancer and left-sided colon cancer have epidemiological, clinical, and molecular biological

(c) The Author(s). 2018 Open Access This article is distributed under the terms of the Creative Commons Attribution 4.0 International License (http://creativecommons.org/licenses/by/4.0/), which permits unrestricted use, distribution, and reproduction in any medium, provided you give appropriate credit to the original author(s) and the source, provide a link to the Creative Commons license, and indicate if changes were made. The Creative Commons Public Domain Dedication waiver (http://creativecommons.org/publicdomain/zero/1.0/) applies to the data made available in this article, unless otherwise stated. 
differences [8-10]. Right-sided colon cancer is more likely to belong to the consensus molecular subtype 2 (CMS2) and to show high frequency of DNA somatic copy number alterations (SCNA) and microsatellite stable/weak immune activation, which makes it relatively insensitive to immunotherapy [11]. It has even been suggested that right-sided colon cancer and left-sided colon cancer may be two different entities. Thus the recommendation that a minimum of 12 lymph nodesbe evaluated may not be equally applicable in right-sided colon cancer and left-sided colon cancer though, so far, there is little biologic evidence to support this theory.

The lymph node ratio-defined as number of positive lymph nodes / the number of total lymph nodes evaluated-independently predicts prognosis in stage III colon cancer [12] and may thus act as a confounder. Wang et al. found that the significance of the total number of lymph nodes as a quality-of-care measure in stage III colon cancer disappeared after adjusting for the effect of lymph nodes ratio [13]. However, they did not take tumor location into consideration. Additionally, their sample included patients treated from 1998 to 2003. Whether their conclusion remains valid in patients treated after 2003 is not known. Especially when a previous study has reported that between 1988 and 2011 there has been marked increase (from $20 \%$ to $80 \%$ ) in the number of patients having $\geq 12$ lymph nodes excised during colectomy for colon cancer [14].

Whether the lymph node ratio has the same confounding influence in both left-sided colon cancer and right-sided colon cancer remains unknown. The aim of this study was to evaluate whether the recommended standard of $\geq 12$ for total number of lymph nodes has the same prognostic significance in right-sided colon cancer and left-sided colon cancer after adjusting for the lymph nodes ratio in a large national cohort.

\section{Methods}

The data were obtained from the Surveillance, Epidemiology, and End Results (SEER) cancer registry. All patients receiving a first diagnosis of invasive stage III colon cancer (according to the 6th the American Joint Committee of cancer) criteria during the period from January 2004 through December 2012 were identified from the registry. The TNM stage was determined by SEER's "extent of disease" (for $\mathrm{T}$ and $\mathrm{M}$ stage) and the "number of positive nodes" (for N stage) coding schemes. All patients had pathologically confirmed adenocarcinoma or mucinous adenocarcinoma [15], with tumor grade categorized as well differentiated, moderately differentiated, poorly differentiated, or undifferentiated. Colon cancer was evaluated as right-sided colon cancer (cecum, ascending colon, hepatic flexure, and transverse colon) or left-sided colon cancer (splenic flexure, descending colon, and sigmoid colon).
The number of positive lymph node and the total number of lymph nodes evaluated were recorded, and lymph nodes ratio was calculated. Race/ethnicity was categorized as previously described. [16]

Patients were observed from 6 months after first diagnosis of colon cancer until the last follow-up, death, or end of the study, whichever occurred first.

\section{Statistical analysis}

Patients were separated into four groups according to lymph nodes ratio value [12] as follows: lymph nodes ratio $1,<0.07$; lymph nodes ratio $2,0.07-0.25$, lymph nodes ratio $3,0.25-0.50$, and lymph nodes ratio $4,0.50$ 1.0. Patients were also grouped according to total number of lymph nodes into high total number of lymph nodes $(\geq 12)$ and low total number of lymph nodes $(<12)$ groups. Multivariate Cox proportional hazard model was used to evaluate the prognostic significance of total number of lymph nodes in left-sided colon cancer and right-sided colon cancer before and after adjustment for lymph nodes ratio. Kaplan-Meier analysis was used to estimate the survival difference between the different total number of lymph nodes and lymph nodes ratio subgroups. The optimal cutoff level for total number of lymph nodes was determined using the web-based application Cutoff Finder (http://medicine.yale.edu/lab/rimm/ research/software.aspx) [17]. The data are presented in a triangular grid and each point represents a cut off value. The strength of the association of each cut-off point is reflected by the intensity of the color. We can move the cursor across the grid and the X-tile software allow the user to move a cursor across the grid and acquire the histogram of the resulting population subsets along with an associated Kaplan Meier curve. Then we can get the minimum $P$ values from log-rank $\chi 2$ statistics for the total number of lymph nodes in terms of survival using the X-tile $[18,19]$.

Two-sided $P<0.05$ was considered statistically significant. Statistical analysis was performed using SAS 9.2 (SAS Institute, Cary, NC, USA) and the survival package within R 2.11 (http://www.r-project.org).

\section{Results \\ Patient characteristics}

Totally, 63,243 stage III patients with colon cancer were identified in the SEER database. Among these patients, 39,024 could be classified as right-sided colon cancer and 24,219 as left-sided colon cancer. The present study included 30, 433 (48.1\%)men and 32,810 (51.9\%) women, ranging 19-82 years (median age: 69 years). 16,127 (25\%) had $<12$ lymph nodes excised (low total number of lymph nodes) and 47,116 (75\%) had $\geq 12$ lymph nodes excised (high total number of lymph). The proportion of high total number of lymph nodes patients was higher in 
right-sided colon cancer than in left-sided colon cancer (79\% vs. $68 \%$; Table 1$)$.

The total number of lymph nodes ranged from 1 to 90 in the entire cohort, with a median of 17. Median total number of lymph nodes was 18 and 15, respectively, in right-sided colon cancer and left-sided colon cancer. The proportion of patients with $1,2-3,4-6$, or $\geq 7$ positive nodes was comparable in the high total number of lymph nodes and low total number of lymph nodes groups. In addition, the proportion of patients with definitely positive lymph nodes was similar in left-sided colon cancer and right-sided colon cancer.

Total number of lymph nodes $<12$ and $\geq 12$ were seen in $3 \%$ vs. $31 \%$ patients in lymph nodes ratio1; $44 \%$ vs. $43 \%$ patients in lymph nodes ratio $2 ; 29 \%$ vs. $17 \%$ patients in lymph nodes ratio 2 ; and $25 \%$ vs. $10 \%$ patients in lymph nodes ratio 4 . The difference between the proportions of low total number of lymph nodes and high total number of lymph nodes was significant in lymph nodes ratio 1 , lymph nodes ratio 3 , and lymph nodes ratio 4. Correspondingly, there were significant difference between the proportions of lymph nodes ratiol, lymph nodes ratio 3 , and lymph nodes ratio 4 in the high total number of lymph nodes and low total number of lymph nodes groups in both right-sided colon cancer and leftsided Patients with colon cancer. Table 1 shows the likelihood of having the recommended 12 lymph nodes excised, classified by clinical feature and demographic characteristic.

\section{Univariate and multivariate cox regression analysis}

The univariate Cox proportional hazard model showed that high total number of lymph nodes patients had a $24 \%$ lower probability of death than low total number of lymph nodes patients patients ( $\mathrm{HR}=0.758,95 \% \mathrm{CI}, 0.739-0.778$; $P<0.001$ ) (Table 2). In multivariate Cox regression analysis high total number of lymph nodes patients patients had $25 \%$ lower probability of death $(\mathrm{HR}=0.747,95 \% \mathrm{CI}$, $0.728-0.726 ; P<0.001$ ) when no adjustment was made for lymph nodes ratio (Table 3). Interestingly, however, after adjusting for lymph nodes ratio, high total number of lymph nodes patients had a $6 \%$ higher probability of death than low total number of lymph nodes patients $(\mathrm{HR}=0$. 938, 95\% CI, 0.909-0.967; $P<0.001$ ).

In univariate analysis, the probability of death was lower in high total number of lymph nodes patients than in low total number of lymph nodes patients in both right-seided colon cancer (lower by $31 \%$ ) and left-sided colon cancer (lower by 23\%) (Table 2). In multivariate Cox regression without adjusting for lymph nodes ratio, these figures were still relatively high $(29 \%$ and $22 \%$ in right-sided colon cancer and left-sided colon cancer, respectively) (Table 3). After adjusting for lymph nodes ratio, the survival advantage of high total number of lymph nodes disappeared in left-sided colon cancer but persisted in right-sided colon cancer where, however, the survival advantage was now only $3 \%$ higher for high total number of lymph nodes patients.

To avoid the influence of using the empirical cutoff points for the stratification of lymph nodes ratio and total number of lymph nodes, the previous analysis was repeated by utilizing using both lymph nodes ratio and total number of lymph nodes both as continuous variables. In the univariate Cox regression, for each unit increase in total number of lymph nodes the probability of death decreased by $1 \%, 1 \%$, and $1 \%$, respectively, in the whole cohort, the right-sided colon cancer cohort, and the left-sided colon cancer cohort (Table 2). In the univariate Cox mode, the lower chance of death for each additional total number of lymph nodes in a patient were $1 \%, 1 \%$ and $1 \%$ for the whole cohort, the rightsided colon cancer cohort and the left-sided colon cancer cohort $(\mathrm{HR}=0.985,95 \% \mathrm{CI}=0.983-0.986, P<0.001$; $\mathrm{HR}=0.90,0.978-0.982, P<0.001 ; \mathrm{HR}=0.986,95 \% \mathrm{CI}, 0$. 983-0.988, $P<0.001$; respectively) (Table 3 ). Paradoxically, after the adjustment for lymph nodes ratio, a patient has $0.4 \%$ higher chance probability of death for each additional unit increase in total number of lymph nodes (HR $=1.004,95 \% \mathrm{CI}, 1.003-1.006 ; P<0.0001$ ) (Table 3). In multivariate analysis, total number of lymph nodes was a predictor of survival, irrespective of whether or not adjustment was made for lymph nodes ratio.

\section{Optimal cutoff for total number of lymph nodes in left-sided colon cancer}

Using the Cutoff Finder tool, we determined the optimal cutoff for total number of lymph nodes in left-sided colon cancer to be 11; this value provided the greatest separation of the OS curves in Kaplan-Meier analysis (Fig. 1). Univariate analysis showed that in the left-sided colon cancer cohort, patients with total number of lymph nodes $\geq 11$ had $26 \%$ greater probability of survival (HR $=0.742$, 95\% CI, 0.706-0.779; $P<0.001)$ than patients with total number of lymph nodes $<11$. In the multivariate Cox regression, the higher probability of survival persisted, irrespective of whether or not adjustment was made for lymph nodes ratio $(\mathrm{HR}=0.704,95 \%$ CI, 0.669-0.740; $P<0.001$; without adjustment for lymph nodes ratio and $\mathrm{HR}=0.868,95 \% \mathrm{CI}, 0.813-0.927 ; P<0$. 001; after adjustment for lymph nodes ratio).

\section{Survival comparison}

The 5-year survival rate was 59\% in the high total number of lymph nodes subgroup, which was 1.2 times higher than that in the low total number of lymph nodes subgroup (Fig. 2a). Survival was better in high total number of lymph nodes patients in both right-sided colon cancer and left-sided colon cancer (Additional file 1: Figure S1A 
Table 1 Clinical characteristics of patients in the full cohort, the Right-sided cohort, and the Left-sided cohort

\begin{tabular}{|c|c|c|c|c|c|c|}
\hline \multirow[t]{2}{*}{ Patients characteristics } & \multicolumn{2}{|l|}{$\begin{array}{l}\text { Full cohort } \\
(N=63,243\end{array}$} & \multicolumn{2}{|l|}{$\begin{array}{l}\text { Right-sided } \\
(N=39,024)\end{array}$} & \multicolumn{2}{|l|}{$\begin{array}{l}\text { Left-sided } \\
(N=24,219)\end{array}$} \\
\hline & $\begin{array}{l}\text { Total number of } \\
\text { lymph nodes }<12\end{array}$ & $\begin{array}{l}\text { Total number of } \\
\text { lymph nodes } \geq 12\end{array}$ & $\begin{array}{l}\text { Total number of } \\
\text { lymph nodes }<12\end{array}$ & $\begin{array}{l}\text { Total number of } \\
\text { lymph nodes } \geq 12\end{array}$ & $\begin{array}{l}\text { Total number of } \\
\text { lymph nodes }<12\end{array}$ & $\begin{array}{l}\text { Total number of } \\
\text { lymph nodes } \geq 12\end{array}$ \\
\hline & $16,127(25.5 \%)$ & $47,116(74.5 \%)$ & $8370(21.4 \%)$ & $30,654(78.6 \%)$ & $7757(32.0 \%)$ & $16,462(68.0 \%)$ \\
\hline \multicolumn{7}{|l|}{ Age at diagnosis } \\
\hline$<40$ & $277(1.7 \%)$ & $1800(3.8 \%)$ & $93(1.1 \%)$ & 885 (2.9\%) & $184(2.4 \%)$ & $915(5.6 \%)$ \\
\hline $40-49$ & $872(5.4 \%)$ & $3631(7.7 \%)$ & 291 (3.5\%) & $1792(5.8 \%)$ & $581(7.5 \%)$ & $1839(11.2 \%)$ \\
\hline $50-59$ & $2473(15.3 \%)$ & $8658(18.4 \%)$ & 889 (10.6\%) & $4658(15.2 \%)$ & $1584(20.4 \%)$ & $4000(24.3 \%)$ \\
\hline $60-69$ & 3664 (22.7\%) & $10,934(23.2 \%)$ & 1719 (20.5\%) & $6984(22.8 \%)$ & 1945 (25.1\%) & $3950(24.0 \%)$ \\
\hline$>70$ & 8841 (54.8\%) & 22,093 (46.9\%) & $5378(64.3 \%)$ & 16,335 (53.3\%) & $3463(44.6 \%)$ & $5758(35.0 \%)$ \\
\hline \multicolumn{7}{|l|}{ Gender } \\
\hline Male & 8011 (49.7\%) & $22,422(47.6 \%)$ & 3828 (45.7\%) & $13,797(45.0 \%)$ & $4183(53.9 \%)$ & 8625 (52.4\%) \\
\hline Female & $8116(47.6 \%)$ & 24,694 (52.4\%) & $4542(54.3 \%)$ & $16,857(55.0 \%)$ & $3574(46.1 \%)$ & 7837 (47.6\%) \\
\hline \multicolumn{7}{|l|}{ Race } \\
\hline Black & 2144 (13.3\%) & $5796(12.3 \%)$ & $1113(13.3 \%)$ & 3805 (12.4\%) & $1031(34.1 \%)$ & 1991 (12.1\%) \\
\hline White & $12,446(77.2 \%)$ & $37,081(78.7 \%)$ & 6691 (79.9\%) & $24,651(80.4 \%)$ & $5755(74.2 \%)$ & $12,430(75.5 \%)$ \\
\hline Hispanic/Latino & $119(0.7 \%)$ & $296(0.6 \%)$ & $69(0.8 \%)$ & $181(0.6 \%)$ & $50(0.6 \%)$ & $115(0.7 \%)$ \\
\hline $\begin{array}{l}\text { Asian or Pacific } \\
\text { Islander and others }\end{array}$ & $1378(8.5 \%)$ & $3784(8.0 \%)$ & $484(5.8 \%)$ & $1922(6.3 \%)$ & 894 (11.5\%) & $1862(11.3 \%)$ \\
\hline Unknown & $40(0.2 \%)$ & $159(0.3 \%)$ & $13(0.2 \%)$ & $95(0.3 \%)$ & $27(0.3 \%)$ & $64(0.4 \%)$ \\
\hline \multicolumn{7}{|l|}{ Hispanic } \\
\hline No & $14,460(89.7 \%)$ & 42,480 (90.2\%) & 7579 (90.5\%) & $27,800(90.7 \%)$ & $6881(88.7 \%)$ & 14,680 (89.2\%) \\
\hline Yes & 1667 (10.3\%) & $4636(9.8 \%)$ & 791 (9.5\%) & 2854 (9.3\%) & 876 (11.3\%) & 1782 (10.8\%) \\
\hline \multicolumn{7}{|l|}{ T stage } \\
\hline T0-T2 $(0,2,3)$ & 2723 (16.9\%) & $5553(11.8 \%)$ & 1144 (13.7\%) & 3437 (11.2\%) & 1579 (20.4\%) & 2116 (12.9\%) \\
\hline T3-T4 $(4,5)$ & $13,376(82.9 \%)$ & $41,503(88.1 \%)$ & 7216 (86.2\%) & $27,181(88.7 \%)$ & $6160(79.4 \%)$ & $14,322(87.0 \%)$ \\
\hline $\operatorname{TX}(6)$ & $28(0.2 \%)$ & $60(0.1 \%)$ & $10(0.1 \%)$ & $36(0.1 \%)$ & $18(0.2 \%)$ & $24(0.1 \%)$ \\
\hline \multicolumn{7}{|l|}{ N stage } \\
\hline N1 & 12,005 (74.4\%) & $29,678(63.0 \%)$ & 6057 (72.4\%) & 19,100 (62.3\%) & $5948(76.7 \%)$ & 1809 (23.3\%) \\
\hline $\mathrm{N} 2$ & 4122 (25.6\%) & 17,438 (37.0\%) & $2313(27.6 \%)$ & $11,554(37.7 \%)$ & $10,578(64.3 \%)$ & $5884(35.7 \%)$ \\
\hline \multicolumn{7}{|l|}{ Chemotherapy } \\
\hline No & 8200 (50.8\%) & 19,558 (41.5\%) & 4640 (55.4\%) & 13,549 (44.6\%) & 3560 (45.9\%) & 6009 (36.5\%) \\
\hline Yes & 7927 (49.2\%) & $27,558(58.5 \%)$ & $3730(44.6 \%)$ & 17,105 (55.8\%) & 4197 (54.1\%) & $10,453(63.5 \%)$ \\
\hline \multicolumn{7}{|l|}{ Radiotherapy } \\
\hline No & $37(0.2 \%)$ & $88(0.2 \%)$ & $18(0.2 \%)$ & $57(0.2 \%)$ & 19 (0.2\%) & $31(0.2 \%)$ \\
\hline Yes & $343(2.1 \%)$ & $868(1.8 \%)$ & 107 (1.3\%) & $298(1.0 \%)$ & $236(3.0 \%)$ & $570(3.5 \%)$ \\
\hline Unknown & $15,747(97.6 \%)$ & 46,160 (98.0\%) & 8245 (98.5\%) & 30,299 (98.8\%) & 7502 (96.7\%) & 15,861 (96.3\%) \\
\hline \multicolumn{7}{|l|}{ Marital status } \\
\hline $\begin{array}{l}\text { Single, separated, } \\
\text { divorced, }\end{array}$ & 3574 (22.2\%) & $11,237(23.8 \%)$ & 1734 (20.7\%) & $6932(22.6 \%)$ & $1840(23.7 \%)$ & 4305 (26.2\%) \\
\hline $\begin{array}{l}\text { Married (including } \\
\text { common law) }\end{array}$ & $8568(53.1 \%)$ & $25,176(53.4 \%)$ & $4223(50.5 \%)$ & 15,937 (52.0\%) & $4345(56.0 \%)$ & $9239(56.1 \%)$ \\
\hline Widowed & 3362 (20.8\%) & 8714 (18.5\%) & $2053(24.5 \%)$ & 6469 (21.1\%) & 1309 (16.9\%) & $2245(13.6 \%)$ \\
\hline Unknown & 623 (3.9\%) & 1989 (4.2\%) & 360 (4.3\%) & $1316(4.3 \%)$ & 263 (3.4\%) & 673 (4.1\%) \\
\hline
\end{tabular}


Table 1 Clinical characteristics of patients in the full cohort, the Right-sided cohort, and the Left-sided cohort (Continued)

\begin{tabular}{|c|c|c|c|c|c|c|}
\hline \multirow[t]{2}{*}{ Patients characteristics } & \multicolumn{2}{|l|}{$\begin{array}{l}\text { Full cohort } \\
(N=63,243\end{array}$} & \multicolumn{2}{|l|}{$\begin{array}{l}\text { Right-sided } \\
(N=39,024)\end{array}$} & \multicolumn{2}{|l|}{$\begin{array}{l}\text { Left-sided } \\
(N=24,219)\end{array}$} \\
\hline & $\begin{array}{l}\text { Total number of } \\
\text { lymph nodes }<12\end{array}$ & $\begin{array}{l}\text { Total number of } \\
\text { lymph nodes } \geq 12\end{array}$ & $\begin{array}{l}\text { Total number of } \\
\text { lymph nodes }<12\end{array}$ & $\begin{array}{l}\text { Total number of } \\
\text { lymph nodes } \geq 12\end{array}$ & $\begin{array}{l}\text { Total number of } \\
\text { lymph nodes }<12\end{array}$ & $\begin{array}{l}\text { Total number of } \\
\text { lymph nodes } \geq 12\end{array}$ \\
\hline \multicolumn{7}{|l|}{ Pathology grade } \\
\hline Well-differentiated & 999 (6.2\%) & $2440(5.2 \%)$ & $483(5.8 \%)$ & $1539(5.0 \%)$ & $516(6.7 \%)$ & 901 (5.5\%) \\
\hline $\begin{array}{l}\text { Moderately } \\
\text { differentiated }\end{array}$ & $10.677(66.2 \%)$ & 29,917 (63.5\%) & $5042(60.2 \%)$ & 18,005 (58.7\%) & $5635(72.6 \%)$ & $11,912(72.4 \%)$ \\
\hline Poorly differentiated & 3619 (22.4\%) & $11,956(25.4 \%)$ & 2338 (27.9\%) & 8995 (29.3\%) & $1281(16.5 \%)$ & $2961(18.0 \%)$ \\
\hline Undifferentiated & $401(2.5 \%)$ & $1802(3.8 \%)$ & $274(3.3 \%)$ & $1431(4.7 \%)$ & $127(1.6 \%)$ & $371(2.3 \%)$ \\
\hline Unknown & $431(2.7 \%)$ & $1.001(2.1 \%)$ & $233(2.8 \%)$ & $684(2.2 \%)$ & $198(2.6 \%)$ & 317 (1.9\%) \\
\hline \multicolumn{7}{|l|}{ Positive lymph node } \\
\hline 0 & $421(2.6 \%)$ & $971(2.1 \%)$ & $193(2.3 \%)$ & $576(1.9 \%)$ & $228(2.9 \%)$ & $395(2.4 \%)$ \\
\hline 1 & $6032(37.4 \%)$ & $14,198(30.1 \%)$ & 3050 (36.4\%) & 9165 (29.9\%) & 2982 (38.4\%) & $5033(30.6 \%)$ \\
\hline $2-3$ & 5483 (34.0\%) & $14,244(30.2 \%)$ & $2776(33.2 \%)$ & 9176 (29.9\%) & 2707 (34.9\%) & $5068(30.8 \%)$ \\
\hline $4-6$ & 3023 (18.7\%) & $9226(19.6 \%)$ & $1621(19.4 \%)$ & $6008(19.6 \%)$ & 1402 (18.1\%) & $3218(19.5 \%)$ \\
\hline$\geq 7$ & $1168(7.2 \%)$ & 8477 (18.0\%) & $730(8.7 \%)$ & $5729(18.7 \%)$ & $438(5.6 \%)$ & $2748(16.7 \%)$ \\
\hline \multicolumn{7}{|l|}{ Lymph nodes ratio } \\
\hline$<0.07$ & $421(2.6 \%)$ & $14,484(30.7 \%)$ & $193(2.3 \%)$ & $9518(31.0 \%)$ & $228(2.9 \%)$ & $4966(30.2 \%)$ \\
\hline $0.07-0.25$ & 7089 (44.0\%) & 20,189 (42.8\%) & 3673 (43.9\%) & 12,965 (42.3\%) & 3416 (44.0\%) & 7224 (43.9\%) \\
\hline $0.25-0.50$ & 4645 (28.8\%) & 7954 (16.9\%) & 2361 (28.2\%) & 5172 (16.9\%) & $2284(29.4 \%)$ & 2782 (16.9\%) \\
\hline $0.50-1.0$ & 3972 (24.6\%) & 4489 (9.5\%) & $2143(25.6 \%)$ & 2999 (9.8\%) & 1829 (23.6\%) & $1490(9.1 \%)$ \\
\hline \multicolumn{7}{|l|}{ Tumor site } \\
\hline Right-sided & 8370 (51.9\%) & $30,654(65.1 \%)$ & & & & \\
\hline Left-sided & 7757 (48.1\%) & 16,462 (34.9\%) & & & & \\
\hline
\end{tabular}

and Additional file 2: Figure S2A for right-sided colon cancer and left-sided colon cancer, respectively). The 5year survival in each lymph nodes ratio subgroup was as follows: $65 \%$ in lymph nodes ratio $1,56 \%$ in lymph nodes ratio $2,45 \%$ in lymph nodes ratio 3 , and $30 \%$ in lymph nodes ratio 4 ; the difference between the groups was statistically significant $(P<0.001 ;$ Fig. $2 b)$. The survival differences between the lymph nodes ratio strata were statistically significant in both right-sided colon cancer and left-sided colon cancer $(P<0.0001$; Additional file 1: Figure S1B and Additional file 2: Figure S2B for right-sided colon cancer and left-sided colon cancer, respectively).

An unexpected finding from Kaplan-Meier survival analysis was that high total number of lymph nodes was associated with significantly better survival in the lymph nodes ratio $<0.25$ subgroup but not in the higher lymph nodes ratio subgroups (Fig. 2c-f,low total number od lymph nodes vs high total number od lymph nodes, HR $=1.257,95 \% \mathrm{CI}, 1.165-1.357, P<0.001$, for Fig. $2 \mathrm{c}$; HR $=1.074,95 \%$ CI, 1.052-1.096, $=P<0.001$, for Fig. 2d; $\mathrm{HR}=1.006,95 \%$ CI $0.981-1.033, P=0.619$, for Fig. 2e; $\mathrm{HR}=0.956,95 \% \mathrm{CI}, 0.931-0.982, P<0.001$, for Fig. 2f). When assessed in the context of the right-sided colon cancer cohort, however, this difference in mortality also merely appears to be exists in the lymph nodes ratio $<0$. 25 and the high total number of lymph nodes have better survival than the low total number of lymph nodes in the left-sided colon cancer cohort of the strata lymph nodes ratio $<0.5$ with the threshold of lymph nodes of 10 (Additional file 1: Figure S1C-F and Additional file 2: Figure S2C-F for right-sided colon cancer and left-sided colon cancer, respectively).

\section{Discussion}

The relationship between the total number of lymph nodes and outcome of colon cancer has been extensively studied. [1, 20-27] Our finding of improved survival with higher lymph node yield is consistent with previous research [1, 20-23, 25, 27-31]. The mechanism remains unknown, but potential factors include more accurate tumor staging, improved surgical management, and superior quality of pathology service [32]. Some studies have demonstrated that a stronger host immune response [33] and the molecular/biological characteristics of the tumor are related to high total number of lymph nodes, and these too may be responsible for its effect on prognosis [32, 34]. For a long time, the presumed reason 
Table 2 Univariate Cox regression analysis of the whole cohort, the Right-sided cohort, and the Left-sided cohort

\begin{tabular}{|c|c|c|c|c|c|c|}
\hline \multirow[t]{2}{*}{ Patients Characteristics } & \multicolumn{2}{|l|}{ Full cohort } & \multicolumn{2}{|l|}{ Right-sided } & \multicolumn{2}{|l|}{ Left-sided } \\
\hline & $\mathrm{HR}(95 \% \mathrm{Cl})$ & $P$ & $\mathrm{HR}(95 \% \mathrm{Cl})$ & $P$ & $\mathrm{HR}(95 \% \mathrm{Cl})$ & $P$ \\
\hline Age at diagnosis & & $<0.001$ & & $<0.001$ & & $<0.001$ \\
\hline$<40$ & 1.000 & & 1.000 & & 1.000 & \\
\hline $40-49$ & $1.101(0.987-1.229)$ & 0.319 & $1.080(0.928-1.258)$ & 0.319 & $1.126(0.961-1.320)$ & 0.143 \\
\hline $50-59$ & $1.158(1.048-1.279)$ & 0.007 & $1.205(1.051-1.381)$ & 0.007 & $1.086(0.938-1.256)$ & 0.270 \\
\hline $60-69$ & $1.557(1.414-1.716)$ & $<0.001$ & $1.499(1.313-1.711)$ & $<0.001$ & $1.523(1.321-1.757)$ & $<0.001$ \\
\hline$>70$ & $3.364(3.063-3.695)$ & $<0.001$ & $2.999(2.636-3.411)$ & $<0.001$ & $3.646(3.178-4.183)$ & $<0.001$ \\
\hline \multicolumn{7}{|l|}{ Gender } \\
\hline Female vs. Male & 0.989 (0.960-1.019) & 0.018 & 0.475 & 0.875 & $0.839-0.913$ & $<0.001$ \\
\hline Race & & $<0.001$ & & $<0.001$ & & $<0.001$ \\
\hline Black & 1.000 & & 1.000 & & 1.000 & \\
\hline White & $0.994(0.959-1.031)$ & 0.756 & $1.056(1.010-1.105)$ & 0.017 & $0.879(0.827-0.935)$ & $<0.001$ \\
\hline Hispanic/Latino & $0.861(0.730-1.016)$ & 0.077 & $0.913(0.743-1.122)$ & $0 / 388$ & $0.793(0.601-1.046)$ & 0.101 \\
\hline $\begin{array}{l}\text { Asian or Pacific Islander } \\
\text { and others }\end{array}$ & $0.699(0.659-0.742)$ & $<0.001$ & $0.766(0.708-0.828)$ & $<0.001$ & $0.671(0.614-0.734)$ & $<0.001$ \\
\hline Unknown & $0.445(0.320-0.618)$ & $<0.001$ & $0.449(0.295-0.684)$ & $<0.001$ & $0.449(0.265-0.761)$ & 0.003 \\
\hline \multicolumn{7}{|l|}{ Hispanic } \\
\hline Yes vs. No & $0.834(0.799-0.871)$ & $<0.001$ & $0.838(0.793-0.885)$ & $<0.001$ & $0.857(0.798-0.921)$ & $<0.001$ \\
\hline T stage & & $<0.001$ & & $<0.001$ & & $<0.001$ \\
\hline T0-T2 $(0,2,3)$ & 1.000 & & 1.000 & & 1.000 & \\
\hline T3-T4 $(4,5)$ & 1.973 (1.889-2.061) & $<0.001$ & $1.784(1.690-1.883)$ & $<0.001$ & 2.212 & $2.055-2.381$ \\
\hline $\operatorname{TX}(6)$ & 1.315 (0.900-1.922) & 0.157 & $1.188(0.714-1.976)$ & 0.507 & $1.575(0.891-2.786)$ & 0.118 \\
\hline \multicolumn{7}{|l|}{$\mathrm{N}$ stage } \\
\hline N2 vs. N1 & $1.581(1.543-1.620)$ & $<0.001$ & $1.657(1.608-1.707)$ & $<0.001$ & $1.415(1.355-1.477)$ & $<0.001$ \\
\hline \multicolumn{7}{|l|}{ Chemotherapy } \\
\hline Yes vs. No & $0.408(0.398-0.418)$ & $<0.001$ & $0.430(0.417-0.443)$ & $<0.001$ & $0.386(0.370-0.403)$ & $<0.001$ \\
\hline Radiotherapy & & $<0.001$ & & $<0.001$ & & 0.001 \\
\hline No & 1.000 & & 1.000 & & 1.000 & \\
\hline Yes & $0.517(0.410-0.654)$ & $<0.001$ & $0.637(0.469-0.865)$ & 0.004 & $0.508(0.351-0.735)$ & $<0.001$ \\
\hline Unknown & $0.524(0.421-0.651)$ & $<0.001$ & $0.515(0.390-0.680)$ & $<0.001$ & $0.506(0.356-0.721)$ & $<0.001$ \\
\hline Marital status & & $<0.001$ & & $<0.001$ & & $<0.001$ \\
\hline Single, separated, divorced, & 1.000 & & 1.000 & & 1.000 & \\
\hline $\begin{array}{l}\text { Married (including } \\
\text { common law) }\end{array}$ & $0.843(0.818-0.870)$ & $<0.001$ & $0.872(0.839-0.907)$ & $<0.001$ & $0.788(0.748-0.829)$ & $<0.001$ \\
\hline Widowed & $1.681(1.623-1.740)$ & $<0.001$ & $1.592(1.524-1.659)$ & $<0.001$ & $1.746(1.643-1.856)$ & $<0.001$ \\
\hline Unknown & 1.065 (0.996-1.139) & 0.065 & $1.082(0.998-1.173)$ & 0.056 & $0.984(0.873-1.110)$ & 0.792 \\
\hline Pathology grade & & $<0.001$ & & $<0.001$ & & $<0.001$ \\
\hline Well-differentiated & 1.000 & & 1.000 & & 1.000 & \\
\hline Moderately differentiated & 1.119 (1.056-1.186) & $<0.001$ & $1.197(1.112-1.289)$ & $<0.001$ & $1.020(0.930-1.119)$ & 0.669 \\
\hline Poorly differentiated & $1.635(1.540-1.736)$ & $<0.001$ & $1.673(1.551-1.804)$ & $<0.001$ & $1.436(1.300-1.586)$ & $<0.001$ \\
\hline Undifferentiated & $1.981(1.825-2.150)$ & $<0.001$ & 1.988 (1.802-2.192) & $<0.001$ & $1.735(1.476-2.040)$ & $<0.001$ \\
\hline Unknown & 1.308 (1.188-1.439) & $<0.001$ & $1.403(1.247-1.578)$ & $<0.001$ & $1.113(0.942-1.315)$ & 0.208 \\
\hline Positive lymph node & & $<0.001$ & & $<0.001$ & & $<0.001$ \\
\hline 0 & 1.000 & & 1.000 & & 1.000 & \\
\hline 1 & $0.943(0.851-1.045)$ & 0.265 & $0.933(0.819-1.062)$ & 0.293 & $0.932(0.787-1.105)$ & 0.418 \\
\hline
\end{tabular}


Table 2 Univariate Cox regression analysis of the whole cohort, the Right-sided cohort, and the Left-sided cohort (Continued)

\begin{tabular}{|c|c|c|c|c|c|c|}
\hline \multirow[t]{2}{*}{ Patients Characteristics } & \multicolumn{2}{|l|}{ Full cohort } & \multicolumn{2}{|l|}{ Right-sided } & \multicolumn{2}{|l|}{ Left-sided } \\
\hline & $\mathrm{HR}(95 \% \mathrm{Cl})$ & $P$ & $\mathrm{HR}(95 \% \mathrm{Cl})$ & $P$ & $\mathrm{HR}(95 \% \mathrm{Cl})$ & $P$ \\
\hline $2-3$ & 1.085 (0.979-1.202) & 0.121 & $1.082(0.951-1.232)$ & 0.232 & $1.058(0.893-1.253)$ & 0.514 \\
\hline $4-6$ & $1.347(1.215-1.495)$ & $<0.001$ & $1.385(1.216-1.578)$ & $<0.001$ & $1.236(1.041-1.467)$ & 0.015 \\
\hline$\geq 7$ & $2.012(1.814-2.232)$ & $<0.001$ & 2.101 (1.844-2.393) & $<0.001$ & $1.713(1.441-2.035)$ & $<0.001$ \\
\hline Lymph nodes ratio & & $<0.001$ & & $<0.001$ & & $<0.001$ \\
\hline$<0.07$ & 1.000 & & 1.000 & & 1.000 & \\
\hline $0.07-0.25$ & $1.312(1.267-1.360)$ & $<0.001$ & $1.375(1.318-1.435)$ & $<0.001$ & $1.245(1.168-1.327)$ & $<0.001$ \\
\hline $0.25-0.50$ & $1.771(1.704-1.842)$ & $<0.001$ & $1.942(1.853-2.036)$ & $<0.001$ & $1.597(1.490-1.711)$ & $<0.001$ \\
\hline $0.50-1.0$ & $2.873(2.761-2.990)$ & $<0.001$ & $3.302(3.147-3.466)$ & $<0.001$ & $2.446(2.279-2.625)$ & $<0.001$ \\
\hline \multicolumn{7}{|l|}{ Tumor site } \\
\hline Left-sided vs. Right-sided & $0.716(0.698-0.734)$ & $<0.001$ & & & & \\
\hline \multicolumn{7}{|l|}{${ }^{\mathrm{a}}$ Total number of lymph node } \\
\hline$\geq 12$ vs. $<12$ & $0.758(0.739-0.778)$ & $<0.001$ & $0.692(0.670-0.715)$ & $<0.001$ & $0.765(0.733-0.798)$ & $<0.001$ \\
\hline 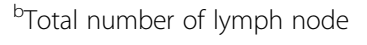 & $0.985(0.983-0.986)$ & $<0.001$ & $0.980(0.978-0.982)$ & $<0.001$ & $0.986(0.983-0.988)$ & $<0.001$ \\
\hline \multicolumn{7}{|l|}{${ }^{\mathrm{C}}$ Total number of lymph nodes } \\
\hline$\geq 11$ vs. $<11$ & & & & & $0.742(0.706-0.779)$ & $<0.001$ \\
\hline
\end{tabular}

Abbreviations: ${ }^{\text {a}}$ Total number of lymph nodes as the Categorical variable

${ }^{\mathrm{b}}$ Total number of lymph nodes as a continuous variable

'The threshold of total number of lymph nodes as 11 in the Left-sided cohort

was that more extensive lymph node evaluation leads to less understaging, and thereby to better treatment and survival. However, LeVoyer et al. [1] and Prandi et al. [35] have all found that increase in the number of lymph nodes retrieved did not necessarily result in upstaging or in increased survival. [36] These authors also showed that the node positivity rate did not change despite a steep increase in total number of lymph nodes, and that it is important to consider the effect of node positivity rate on prognosis. Wong et al. [37] found that distribution of the positive lymph node frequency and the rate of receiving adjuvant chemotherapy are similar among hospital groups although the number of lymph nodes evaluated varied widely, suggesting that increasing total number of lymph nodes does not significantly upstage patients.

In our study, we found all lymph nodes ratio strata were independently predictive of overall survival. Thus, although the 12-node-minimum standard remains an important prognostic determinant, lymph nodes ratio has a separate contributory prognostic role even though the surgical benchmark and lymph nodes ratio are interrelated parameters. Importantly, our study showed no relationship between total number of lymph nodes and outcome in the left-sided colon cancer cohort when adjustment was made for lymph nodes ratio in multivariate analysis; this finding is consistent with Wang et al. [13]

The American Joint Committee on cancer TNM staging system is based on number of positive nodes, not lymph nodes ratio. In the present study, Kaplan-Meier survival analysis showed that high total number of lymph nodes is associated with significantly improved survival in the lymph nodes ratio $<0.25$ subgroup. However, the difference between survival of high total number of lymph nodes and low total number of lymph nodes patients was less in the higher lymph nodes ratio strata. Furthermore, the distribution of lymph nodes ratio strata between the high total number of lymph nodes and low total number of lymph nodes groups was significantly different. This may because low lymph nodes ratio subgroups have lower metastasis risk and fewer positive lymph nodes, and so most of the lymph nodes retrieved during surgery are negative lymph nodes. The fewer number of positive nodes retrieved may cause an artificial inflation of the lymph nodes ratio-the "small-denominator effect." Conversely, the high lymph nodes ratio subgroups have relatively higher metastasis risk and more number of positive lymph nodes. Although more lymph nodes are retrieved in the high total number of lymph nodes, the number of positive lymph nodes is also high, and the tumor burden is the leading role not the immune reaction. In this occasion, a "large-denominator" effect may be an issue as it is much easier to achieve positive nodes sampled.

A threshold total number of lymph nodes of 12 is often used as a measure of quality of surgical care. However, it may not be adequate to only use the number of lymph nodes evaluated as a quality-of-care measure; the surgeon's technique and the pathologist's methods must also be taken into account. Other variables, such as the patient's immunologic response and age [38] and the location of the primary tumor, may also greatly impact the number of lymph nodes retrieved. Prandi et al. have 
Table 3 Multivariate Cox Regression for the whole cohort, the Right-sided cohort and the Left-sided cohort

\begin{tabular}{|c|c|c|c|c|c|c|}
\hline \multirow[t]{2}{*}{ Patients Characteristics } & \multicolumn{2}{|l|}{ Full cohort } & \multicolumn{2}{|l|}{ Right-sided } & \multicolumn{2}{|l|}{ Left-sided } \\
\hline & $\mathrm{HR}(95 \% \mathrm{Cl})$ & $P$ & $\mathrm{HR}(95 \% \mathrm{Cl})$ & $P$ & $\mathrm{HR}(95 \% \mathrm{Cl})$ & $P$ \\
\hline Age at diagnosis & & $<0.001$ & & $<0.001$ & & $<0.001$ \\
\hline$<40$ & 1.000 & & 1.000 & & 1.000 & \\
\hline $40-49$ & $1.074(0.962-1.199)$ & 0.201 & 1.019 (0.875-1.187) & 0.809 & $1.146(0.977-1.343)$ & 0.094 \\
\hline $50-59$ & $1.144(1.035-1.264)$ & .0 .008 & $1.162(1.014-1.333)$ & 0.031 & $1.107(0.956-1.281)$ & 0.174 \\
\hline $60-69$ & $1.466(1.330-1.616)$ & $<0.001$ & $1.422(1.245-1.624)$ & $<0.001$ & $1.462(1.266-1.688)$ & $<0.001$ \\
\hline$>70$ & $2.516(2.287-2.769)$ & $<0.001$ & $2.265(1.987-2.581)$ & $<0.001$ & $2.878(2.500-3.312)$ & $<0.001$ \\
\hline \multicolumn{7}{|l|}{ Sex } \\
\hline Female vs. Male & $0.804(0.784-0.825)$ & $<0.001$ & & & $0.796(0.762-0.833)$ & $<0.001$ \\
\hline Race & & $<0.001$ & & $<0.001$ & & $<0.001$ \\
\hline Black & 1.000 & & 1.000 & & 1.000 & \\
\hline White & $0.866(0.834-0.898)$ & $<0.001$ & $0.907(0.866-0.950)$ & $<0.001$ & 0.797 (0.749-0.848) & $<0.001$ \\
\hline Hispanic/Latino & $0.848(0.718-1.001)$ & 0.051 & $0.856(0.696-1.052)$ & 0.140 & $0.825(0.625-1.090)$ & 0.175 \\
\hline Asian or Pacific Islander and others & $0.687(0.647-0.729)$ & $<0.001$ & $0.699(0.646-0.757)$ & $<0.001$ & $0.663(0.606-0.726)$ & $<0.001$ \\
\hline Unknown & $0.471(0.339-0.654)$ & $<0.001$ & $0.482(0.317-0.734)$ & 0.001 & $0.462(0.273-0.783)$ & 0.004 \\
\hline \multicolumn{7}{|l|}{ Hispanic } \\
\hline Yes vs. No & $0.920(0.881-0.962)$ & $<0.001$ & $0.906(0.857-0.958)$ & $<0.001$ & & \\
\hline T stage & & $<0.001$ & & & & $<0.001$ \\
\hline T0-T2 $(0,2,3)$ & 1.000 & & 1.00 & & 1.000 & \\
\hline T3-T4 $(4,5)$ & $1.703(1.629-1.780)$ & $<0.001$ & $1.540(1.458-1.627)$ & $<0.001$ & $1.984(1.841-2.138)$ & $<0.001$ \\
\hline $\operatorname{TX}(6)$ & $1.366(0.933-1.999)$ & 0.109 & $1.155(0.693-1.926)$ & 0.580 & $1.756(0.991-3.112)$ & 0.054 \\
\hline \multicolumn{7}{|l|}{$\mathrm{N}$ stage } \\
\hline N2 vs. N1 & $1.139(1.097-1.181)$ & $<0.001$ & $1.157(1.105-1.211)$ & $<0.001$ & $1.063(1.005-1.124)$ & 0.033 \\
\hline \multicolumn{7}{|l|}{ Chemotherapy } \\
\hline Yes vs. No & $0.499(0.486-0.513)$ & $<0.001$ & $0.501(0.485-0.517)$ & $<0.001$ & $0.491(0.469-0.513)$ & $<0.001$ \\
\hline Radiotherapy & & & & $<0.001$ & & \\
\hline No & 1.000 & & 1.000 & & 1.000 & \\
\hline Yes & $1.286(1.017-1.626)$ & 0.036 & $1.402(1.031-1.906)$ & 0.031 & $1.253(0.865-1.816)$ & 0.233 \\
\hline Unknown & $0.866(0.696-1.077)$ & 0.196 & $0.851(0.644-1.123)$ & 0.254 & $0.886(0.623-1.262)$ & 0.503 \\
\hline Marital status & & $<0.001$ & & $<0.001$ & & $<0.001$ \\
\hline Single, separated, divorced, & 1.000 & & 1.000 & & 1.000 & \\
\hline Married (including common law) & $0.785(0.761-0.811)$ & $<0.001$ & $0.844(0.811-0.878)$ & $<0.001$ & $0.733(0.695-0.773)$ & $<0.001$ \\
\hline Windowed & $1.078(1.039-1.120)$ & $<0.001$ & $1.051(1.005-1.099)$ & $<0.001$ & $1.029(0.963-1.099)$ & 0.399 \\
\hline Unknown & $0.875(0.818-0.936)$ & $<0.001$ & $0.904(0.834-0.981)$ & 0.015 & $0.812(0.720-0.916)$ & 0.001 \\
\hline Pathology grade & & $<0.001$ & & $<0.001$ & & $<0.001$ \\
\hline Well-differentiated & 1.000 & & 1.000 & & 1.000 & \\
\hline Moderately differentiated & $1.086(1.025-1.151)$ & 0.005 & $1.145(1.063-1.233)$ & $<0.001$ & $1.003(0.915-1.101)$ & 0.942 \\
\hline Poorly differentiated & $1.340(1.262-1.424)$ & $<0.001$ & $1.378(1.276-1.487)$ & $<0.001$ & $1.282(1.160-1.417)$ & $<0.001$ \\
\hline Undifferentiated & $1.636(1.507-1.777)$ & $<0.001$ & $1.658(1.502-1.830)$ & $<0.001$ & 1.639 (1.393-1.928) & $<0.001$ \\
\hline Unknown & $1.228(1.115-1.352)$ & $<0.001$ & 1.275 (1.133-1.435) & $<0.001$ & 1.157 (0.979-1.368) & 0.087 \\
\hline \multicolumn{7}{|l|}{ Tumor site } \\
\hline Left-sided vs. Right-sided & $0.847(0.824-0.870)$ & $<0.001$ & & & & \\
\hline Lymph nodes ratio & & $<0.001$ & & $<0.001$ & & $<0.001$ \\
\hline
\end{tabular}


Table 3 Multivariate Cox Regression for the whole cohort, the Right-sided cohort and the Left-sided cohort (Continued)

\begin{tabular}{|c|c|c|c|c|c|c|}
\hline \multirow[t]{2}{*}{ Patients Characteristics } & \multicolumn{2}{|l|}{ Full cohort } & \multicolumn{2}{|l|}{ Right-sided } & \multicolumn{2}{|l|}{ Left-sided } \\
\hline & HR $(95 \% \mathrm{Cl})$ & $P$ & HR $(95 \% \mathrm{Cl})$ & $P$ & $\mathrm{HR}(95 \% \mathrm{Cl})$ & $P$ \\
\hline $0.07-0.25$ & $1.277(1.229-1.325)$ & $<0.001$ & $1.294(1.236-1.354)$ & $<0.001$ & $1.245(1.167-1.328)$ & $<0.001$ \\
\hline $0.25-0.50$ & $1.602(1.523-1.685)$ & $<0.001$ & $1.663(1.563-1.770)$ & $<0.001$ & $1.514(1.402-1.636)$ & $<0.001$ \\
\hline $0.50-1.0$ & $2.494(2.357-2.640)$ & $<0.001$ & $2.671(2.491-2.863)$ & $<0.001$ & $2.282(2.100-2.480)$ & $<0.001$ \\
\hline \multicolumn{7}{|l|}{$\begin{array}{l}\text { aTotal number of lymph nodes } \\
\text { (without Lymph nodes ratio) }\end{array}$} \\
\hline$\geq 12$ vs. $<12$ & $0.747(0.728-0.766)$ & $<0.001$ & $0.706(0.683-0.730)$ & $<0.001$ & $0.780(0.747-0.815)$ & $<0.001$ \\
\hline \multicolumn{7}{|l|}{$\begin{array}{l}\text { Total number of lymph nodes } \\
\text { (with Lymph nodes ratio) }\end{array}$} \\
\hline$\geq 12$ vs. $<12$ & $0.938(0.909-0.967)$ & $<0.001$ & $0.902(0.886-0.957)$ & $<0.001$ & $0.968(0.919-1.020)$ & 0.223 \\
\hline $\begin{array}{l}\text { 'Total number of lymph nodes } \\
\text { (without Lymph nodes ratio) }\end{array}$ & $1.000(0.998-1.001)$ & 0.694 & $0.981(0.980-0.983)$ & $<0.001$ & $0.985(0.982-0.987)$ & $<0.001$ \\
\hline $\begin{array}{l}\text { Total number of lymph nodes } \\
\text { (with Lymph nodes ratio) }\end{array}$ & $1.004(1.003-1.006)$ & $<0.001$ & $0.994(0.992-0.997)$ & $<0.001$ & $0.996(0.993-1.000)$ & 0.033 \\
\hline \multicolumn{7}{|l|}{$\begin{array}{l}{ }^{ } \text {Total number of lymph nodes } \\
\text { (without Lymph nodes ratio) }\end{array}$} \\
\hline$\geq 11$ vs. $<11$ & & & & & $0.704(0.669-0.740)$ & $<0.001$ \\
\hline \multicolumn{7}{|l|}{$\begin{array}{l}\text { Total number of lymph nodes } \\
\text { (with Lymph nodes ratio) }\end{array}$} \\
\hline$\geq 11$ vs. $<11$ & & & & & $0.868(0.813-0.927)$ & $<0.001$ \\
\hline
\end{tabular}

Abbreviations: ${ }^{\text {a}}$ Total number of lymph nodes as a Categorical variable

${ }^{\mathrm{b}}$ Total number of lymph nodes as a continuous variable

'The threshold is 11 in the Left-sided cohort

shown that the number of lymph nodes retrieved is inversely associated with patient's age [35]. Another study found that more lymph nodes are identified in patients with right-sided colon cancer, which led the authors to question whether the standard for regional lymph nodes evaluation should be the same in right-sided colon cancer and left-sided colon cancer [39].

Our study showed that after adjusting for the lymph nodes ratio, the association of total number of lymph nodes with survival in stage IIII colon cancer was present only in right-sided colon cancer but not in left-sided colon cancer.
The biological behavior of the tumor and host parameters such as immune response may affect the number of traceable lymph nodes. Several authors have hypothesized that smaller numbers of lymph nodes found is reflection of a diminished immune response. Size and morphology of nodes are modified by immune responses, with a weak immune response leading to smaller nodes that are harder to find $[21,25,40]$. One study showed that the number of nodes found is associated with lymphocytic infiltration of the primary tumor, with more nodes being found in patients with prominent lymphocytic infiltration into the primary tumor a

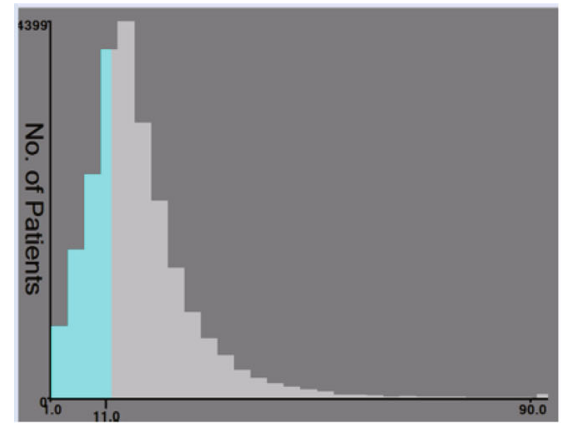

Lymph nodes examined b

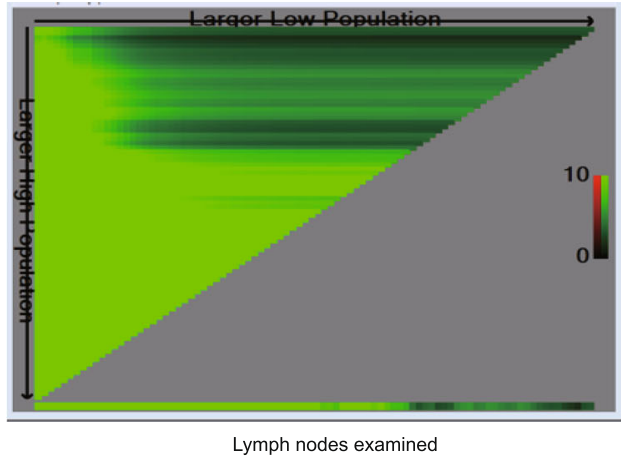

Fig. 1 a The number of lymph nodes distribution according to lymph nodes examined. Ranged from 0 to 90 . $\mathbf{b}$ X-tile plots for lymph nodes constructed by left-sided Patients with colon cancer. The plots dividing them into 2 groups by the cutoff point 10 . The brightest pixel represents the maximum $\mathbf{X}$ log-rank value 


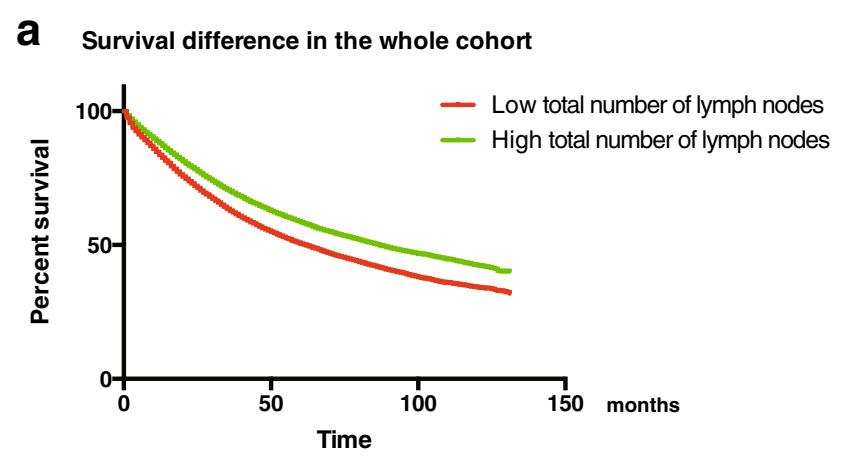

C

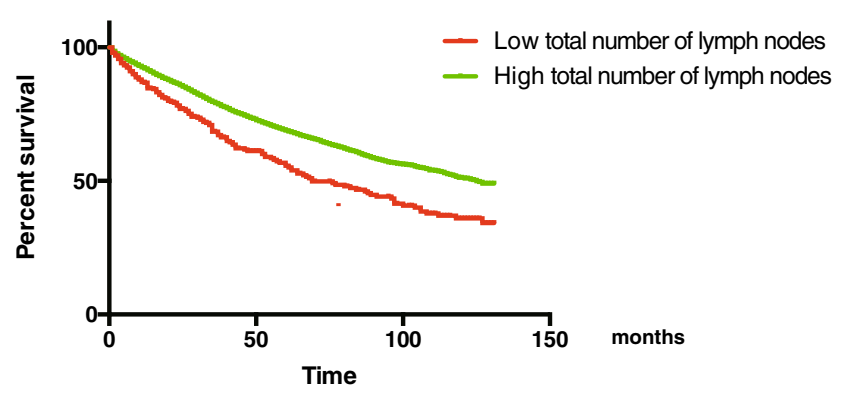

e Survival difference in lymph nodes ratio3

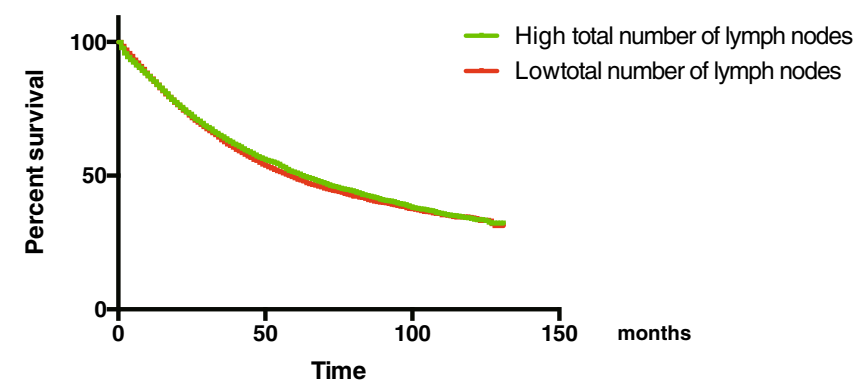

b Survival difference of the lymph nodes ratio in the whole cohort

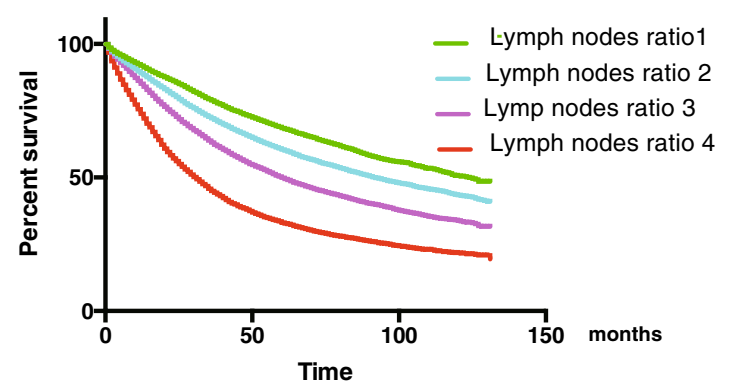

d

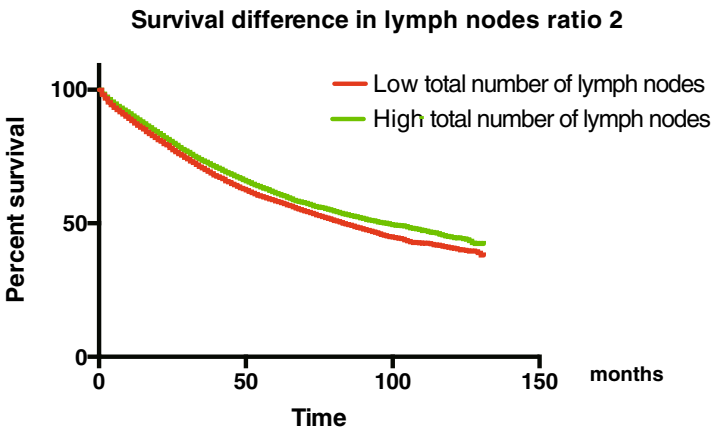

$\mathbf{f}$

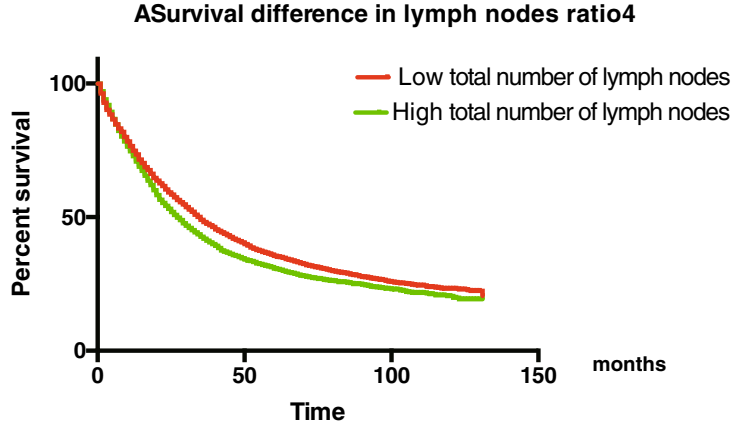

Fig. 2 a Survival curves of patients with low and high total number of lymph nodes. b Survival curves stratified by lymph nodes ratio. c Survival of lymph nodes ratio 1 patients according to total number of lymph nodes. $\mathbf{d}$ Survival of lymph nodes ratio 2 patients according to total number of lymph nodes. e Survival of lymph nodes ratio 3 patients according to total number of lymph nodes. $\mathbf{f}$ Survival of lymph nodes ratio 4 patients according to total number of lymph nodes

than in patients with mild lymphocytic infiltration [41]. The differences in anatomical, physiological, and molecular characteristics between right-sided colon cancer and leftsided colon cancer may also influence the number of lymph nodes examined. [13] The benefits associated with high total number of lymph nodes might actually reflect the host lymphocytic reaction to tumor, which is associated with lymph node count $[21,41]$. This phenomenon is commonly observed in lymph nodes draining the cancer. It has been postulated that the right colon mesentery contains a more complex lymphatic system, which leads to an enhanced immune response and thereby to increase in the number of lymph nodes examined in right-sided colon cancer, but there is no clear evidence in support of this theory [42]. A cadaveric study may help clarify this issue.

\section{Conclusion}

In summary, the study demonstrated that it may not be correct to use the threshold of total number of lymph nodes $\geq 12$ for predicting prognosis in both left-sided colon cancer and right-sided colon cancer in stage III patients with colon cancer. Although the number of lymph nodes evaluated is a prognostic factor, this does not mean that it is also a predictive factor [43]. Presently, many undersampled patients receive unnecessary treatment, and so adequate nodal evaluation is important. There is little evidence to justify adjuvant treatment in node-negative patients when too few nodes are evaluated. Based on the results of the present study, the surgeon should take tumor location into consideration when deciding on the minimum total number of lymph nodes to be retrieved. 


\section{Additional files}

Additional file 1: Figure. S1. (A) Survival curves for low- and high-total number of lymph nodes patients in the left-sided colon cancer cohort. (B) Survival curves stratified by lymph nodes ratio in the left-sided colon cancer cohort. (C) Survival of lymph nodes ratio 1 patients according to total number of lymph nodes in the left-sided colon cancer cohort. (D) Survival of lymph nodes ratio 2 patients according to total number of lymph nodes in the left-sided colon cancer cohort. (E) Survival of lymph nodes ratio 3 patients according to total number of lymph nodes in the left-sided colon cancer cohort. (F) survival of lymph nodes ratio 4 patients according to total number of lymph nodes in the left-sided colon cancer cohort. (PDF 1077 kb)

Additional file 2: Figure S2. (A) Survival curves of low- and high- total number of lymph nodes patients in the right-sided colon cancer cohort. (B) Survival curves stratified by lymph nodes ratio in the right-sided colon cancer cohort. (C) Survival of lymph nodes ratio 1 patients according to total number of lymph nodes in the right-sided colon cancer cohort. (D) Survival of lymph nodes ratio 2 patients according to total number of lymph nodes in the right-sided colon cancer cohort; (E) Survival of lymph nodes ratio 3 patients according to total number of lymph nodes in the right-sided colon cancer cohort. (F) Survival of lymph nodes ratio 4 patients according to total number of lymph nodes in the right-sided colon cancer cohort. (PDF $1068 \mathrm{~kb}$ )

\section{Abbreviation}

SEER: Surveillance Epidemiology and End Results

\section{Acknowledgements}

We would like to thank the native English speaking scientists of Elixigen Company (Huntington Beach, California) for editing our manuscript.

\section{Funding}

This work was supported by the Natural Science Foundation of Guangdong, China [grant number 2015A030313010].

\section{Availability of data and materials}

All data were retrieved from the Surveillance, Epidemiology, and End Results (SEER) program of the National Cancer Institute between 2004 and 2012. The datasets are available in the SEER dataset repository https://seer.cancer.gov/.

\section{Authors' contributions}

$L Y, Z X$, and LX made substantial contributions to study conception and design; $L Y, Z X$ collected the data; $L Y, Z X$ and $L X$ analyzed the data and drafted the manuscript; $Q X, G G, P K, W H, C J, S L$ analyzed the data; $L X$ revised it critically for important intellectual content; LX agreed to be accountable for all aspects of the work in ensuring that questions related to the accuracy or integrity of any part of the work are appropriately investigated and resolved. All authors ( $L Y, Z X, Q X, W H, S L, P K, C J, G G, L X)$ have read and approved the final manuscript.

\section{Ethics approval and consent to participate}

As the data used was from SEER dataset (public), Ethics approval and consent to participate could be checked in SEER.

\section{Competing interests}

All authors declare that they have no competing interests.

\section{Publisher's Note}

Springer Nature remains neutral with regard to jurisdictional claims in published maps and institutional affiliations.

\section{Author details}

'Sun Yat-sen University Cancer Center, 651 Dongfeng Road east, Guangzhou 510060, China. ${ }^{2}$ State Key Laboratory of Oncology in Southern China, Guangzhou, China. ${ }^{3}$ Collaborative Innovation Center for Cancer Medicine, Guangzhou, China.
Received: 9 February 2018 Accepted: 24 April 2018

Published online: 11 May 2018

\section{References}

1. Le Voyer TE, Sigurdson ER, Hanlon AL, et al. Colon cancer survival is associated with increasing number of lymph nodes analyzed: a secondary survey of intergroup trial INT-0089. J Clin Oncol. 2003;21(15):2912-9.

2. Baxter NN, Ricciardi R, Simunovic M, et al. An evaluation of the relationship between lymph node number and staging in pT3 colon cancer using population-based data. Dis Colon rectum. 2010;53(1):65-70.

3. Parsons HM, Tuttle TM, Kuntz KM, et al. Association between lymph node evaluation for colon cancer and node positivity over the past 20 years. JAMA. 2011;306(10):1089-97.

4. Compton C, Fenoglio-Preiser CM, Pettigrew N, et al. American joint committee on Cancer prognostic factors consensus conference: colorectal working group. Cancer. 2000;88(7):1739-57.

5. Yarbro JW, Page DL, Fielding LP, et al. American joint committee on Cancer prognostic factors consensus conference. Cancer. 1999;86(11):2436-46.

6. Li Destri G, Di Carlo I, Scilletta R, et al. Colorectal cancer and lymph nodes: the obsession with the number 12. World J Gastroenterol. 2014;20(8):1951-60.

7. Washington MK, Berlin J, Branton P, et al. Protocol for the examination of specimens from patients with primary carcinoma of the colon and rectum. Arch Pathol Lab Med. 2009;133(10):1539-51.

8. Benedix F, Kube R, Meyer F, et al. Comparison of 17,641 patients with rightand left-sided colon cancer: differences in epidemiology, perioperative course, histology, and survival. Dis Colon rectum. 2010;53(1):57-64.

9. Nawa T, Kato J, Kawamoto $\mathrm{H}$, et al. Differences between right- and leftsided colon cancer in patient characteristics, cancer morphology and histology. J Gastroenterol Hepatol. 2008;23(3):418-23.

10. Elsaleh H, Joseph D, Grieu F, et al. Association of tumour site and sex with survival benefit from adjuvant chemotherapy in colorectal cancer. Lancet. 2000;355(9217):1745-50.

11. Guinney J, Dienstmann $R$, Wang $X$, et al. The consensus molecular subtypes of colorectal cancer. Nat Med. 2015;21(11):1350-6.

12. Wang J, Hassett JM, Dayton MT, et al. Lymph node ratio: role in the staging of node-positive colon cancer. Ann Surg Oncol. 2008;15(6):1600-8.

13. Rosenberg R, Friess $H$. Should total number of lymph nodes be used as a quality of care measure for stage III colon cancer? Ann Surg. 2010;251(1): 183. author reply 184-5

14. Garcia B, Guzman C, Johnson C, et al. Trends in lymph node excision and impact of positive lymph node ratio in patients with colectomy for primary colon adenocarcinoma: population based study 1988 to 2011. Surg Oncol. 2016;25(3):158-63.

15. Ashley J. The international classification of diseases: the structure and content of the tenth revision. Health Trends. 1990:22(4):135-7.

16. Lo NS, Sarr MG. Mucinous cystadenocarcinoma of the appendix. The controversy persists: a review. Hepato-Gastroenterology. 2003;50(50):432-7.

17. Budczies J, Klauschen F, Sinn BV, et al. Cutoff finder: a comprehensive and straightforward web application enabling rapid biomarker cutoff optimization. PLoS One. 2012:7(12):e51862.

18. Hilsenbeck SG, Clark GM, McGuire WL. Why do so many prognostic factors fail to pan out? Breast Cancer Res Treat. 1992;22(3):197-206.

19. Camp RL, Dolled-Filhart M, Rimm DL. X-tile: a new bio-informatics tool for biomarker assessment and outcome-based cut-point optimization. Clin Cancer Res. 2004;10(21):7252-9.

20. Swanson RS, Compton CC, Stewart AK, et al. The prognosis of T3NO colon cancer is dependent on the number of lymph nodes examined. Ann Surg Oncol. 2003;10(1):65-71.

21. Caplin S, Cerottini JP, Bosman FT, et al. For patients with Dukes' B (TNM stage II) colorectal carcinoma, examination of six or fewer lymph nodes is related to poor prognosis. Cancer. 1998;83(4):666-72.

22. Joseph NE, Sigurdson ER, Hanlon AL, et al. Accuracy of determining nodal negativity in colorectal cancer on the basis of the number of nodes retrieved on resection. Ann Surg Oncol. 2003;10(3):213-8.

23. Law CH, Wright FC, Rapanos T, et al. Impact of lymph node retrieval and pathological ultra-staging on the prognosis of stage II colon cancer. J Surg Oncol. 2003:84(3):120-6.

24. Jestin P, Pahlman L, Glimelius B, et al. Cancer staging and survival in colon cancer is dependent on the quality of the pathologists' specimen examination. Eur J Cancer. 2005;41(14):2071-8. 
25. Sarli L, Bader G, lusco D, et al. Number of lymph nodes examined and prognosis of TNM stage II colorectal cancer. Eur J Cancer. 2005:41(2):272-9.

26. Johnson PM, Porter GA, Ricciardi R, et al. Increasing negative lymph node count is independently associated with improved long-term survival in stage IIIB and IIIC colon cancer. J Clin Oncol. 2006;24(22):3570-5.

27. Chen SL, Bilchik AJ. More extensive nodal dissection improves survival for stages I to III of colon cancer: a population-based study. Ann Surg. 2006; 244(4):602-10.

28. Chang GJ, Rodriguez-Bigas MA, Skibber JM, et al. Lymph node evaluation and survival after curative resection of colon cancer: systematic review. J Natl Cancer Inst. 2007:99(6):433-41.

29. Vather R, Sammour T, Kahokehr A, et al. Lymph node evaluation and longterm survival in stage II and stage III colon cancer: a national study. Ann Surg Oncol. 2009;16(3):585-93.

30. Wright $\mathrm{FC}$, Law $\mathrm{CH}$, Berry S, et al. Clinically important aspects of lymph node assessment in colon cancer. J Surg Oncol. 2009;99(4):248-55.

31. Elferink MA, Siesling S, Visser O, et al. Large variation between hospitals and pathology laboratories in lymph node evaluation in colon cancer and its impact on survival, a nationwide population-based study in the Netherlands. Ann Oncol. 2011;22(1):110-7.

32. Ogino S, Nosho K, Irahara N, et al. Negative lymph node count is associated with survival of colorectal cancer patients, independent of tumoral molecular alterations and lymphocytic reaction. Am J Gastroenterol. 2010; 105(2):420-33.

33. Ogino S, Galon J, Fuchs CS, et al. Cancer immunology-analysis of host and tumor factors for personalized medicine. Nat Rev Clin Oncol. 2011;8(12): $711-9$.

34. van Steenbergen LN, Elferink MA, Krijnen P, et al. Improved survival of colon cancer due to improved treatment and detection: a nationwide populationbased study in the Netherlands 1989-2006. Ann Oncol. 2010;21(11):2206-12.

35. Prandi $M$, Lionetto $R$, Bini $A$, et al. Prognostic evaluation of stage $B$ colon cancer patients is improved by an adequate lymphadenectomy: results of a secondary analysis of a large scale adjuvant trial. Ann Surg. 2002;235(4):458-63.

36. Luebke T, Baldus SE, Zirbes TK, et al. Lymph node revealing solution in gastric carcinoma does not provide upstaging of the N-status. Oncol Rep. 2005;13(2):361-5.

37. Wong SL, Ji H, Hollenbeck BK, et al. Hospital lymph node examination rates and survival after resection for colon cancer. JAMA. 2007;298(18):2149-54.

38. Bilimoria KY, Stewart AK, Palis BE, et al. Adequacy and importance of lymph node evaluation for colon cancer in the elderly. J Am Coll Surg. 2008;206(2): $247-54$

39. Guan X, Chen W, Liu Z, et al. Whether regional lymph nodes evaluation should be equally required for both right and left colon cancer. Oncotarget. 2016:7(37):59945-56.

40. Lemmens VE, van Lijnschoten I, Janssen-Heijnen ML, et al. Pathology practice patterns affect lymph node evaluation and outcome of colon cancer: a population-based study. Ann Oncol. 2006;17(12):1803-9.

41. George S, Primrose J, Talbot R, et al. Will Rogers revisited: prospective observational study of survival of 3592 patients with colorectal cancer according to number of nodes examined by pathologists. Br J Cancer. 2006: 95(7):841-7.

42. Bilimoria KY, Palis B, Stewart AK, et al. Impact of tumor location on nodal evaluation for colon cancer. Dis Colon rectum. 2008;51(2):154-61.

43. van Steenbergen $L N$, van Lijnschoten $G$, Rutten $H J$, et al. Improving lymph node detection in colon cancer in community hospitals and their pathology department in southern Netherlands. Eur J Surg Oncol. 2010; 36(2):135-40.

\section{Ready to submit your research? Choose BMC and benefit from:}

- fast, convenient online submission

- thorough peer review by experienced researchers in your field

- rapid publication on acceptance

- support for research data, including large and complex data types

- gold Open Access which fosters wider collaboration and increased citations

- maximum visibility for your research: over $100 \mathrm{M}$ website views per year

At BMC, research is always in progress.

Learn more biomedcentral.com/submissions 